\title{
EL PLAN MAESTRO, DIÁLOGOS PARA LA PROFESIÓN DOCENTE
}

\author{
Hernán Hochschild, Facundo Díaz, Joaquín Walker, \\ Jorge Schiappacasse y María Paz Medeiros ${ }^{1}$
}

\begin{abstract}
RESUMEN
El siguiente documento sistematiza la experiencia de "El Plan Maestro", su proceso de creación, la metodología desarrollada y las principales propuestas generadas. El Plan Maestro se constituyó como una agrupación de 20 instituciones diversas que creó una plataforma de participación ciudadana para desarrollar, en 120 días, propuestas de política pública que incidieran en la discusión de la política docente. El proceso contempló distintas instancias de participación, donde los actores dialogaron respecto de los temas que afectan hoy a los educadores y educadoras y la necesidad de dar urgencia y prioridad nacional a la temática docente en el contexto de las reformas educativas en discusión. En este artículo se rescatan los doce principios orientadores de la política nacional docente que surgen desde los espacios de diálogo entre los actores. Estos, junto con 130 propuestas, buscan sentar las bases para el desarrollo de una profesión docente y mejorar las condiciones de formación y de enseñanza de los educadores y educadoras de Chile, con el fin de alcanzar mayor calidad y equidad en la educación de todos los niños, niñas y jóvenes de Chile.
\end{abstract}

Palabras clave: políticas docentes, participación ciudadana, políticas públicas, profesión docente.

\section{PLAN MAESTRO, DIALOGUES FOR THE TEACHING PROFESSION}

\section{ABSTRACT}

The following document systematizes the experience of "El Plan Maestro", its creation process, the methodology developed and the main proposals generated. "El Plan Maestro" was formed by a group of 20 diverse institutions that created a platform for citizen participation with the objective to develop -within 120 days-public policy proposals that would influence the discussion of teacher related policies. The process included different levels of participation in which various actors discussed topics that affect today's teachers and the need to make the topic of teaching a national priority, in light of the current discussion of educational reforms. This article delineates the twelve guiding principles for national teaching policy that emerged from these conversations. Said principles, along with 130 proposals, seek to lay the groundwork for the development of the teaching profession, and to improve the preparation and teaching conditions for educators in Chile, with the goal of achieving greater quality and equity in education for all of Chile's children.

Keywords: teacher related policies, citizen participation, public policy, teaching profession.

1 Todos los autores forman parte de la iniciativa "Elige Educar", Santiago, Chile. Contacto: hhochschild@eligeeducar.cl 


\section{La profesión docente en Chile y los inicios de El Plan Maestro}

A lo largo de las últimas décadas la evidencia internacional ha demostrado la importancia que tienen los profesores en el logro de los aprendizajes y en el desarrollo de habilidades de los niños, niñas y jóvenes al interior de las escuelas (Sanders \& Rivers, 1996; Rockoff, 2004; Rivkin, Hanushek \& Kain, 2005). Esto ha abierto el debate a nivel mundial respecto de la necesidad de impulsar políticas que busquen atraer, formar y retener a los buenos profesores en el sistema, particularmente en las salas de clases, con el objetivo de alcanzar una educación de calidad (OECD, 2005).

Países como Finlandia y Singapur, entre otros, que han avanzado significativamente en términos de logro de resultados en los aprendizajes de sus alumnos, han focalizado sus recursos en políticas docentes (García et. al, 2013), mientras que en Chile ha ocurrido lo contrario: las condiciones de la profesión docente han favorecido que esta se haya ido desvalorizando a lo largo de los años, afectando la percepción que tiene la sociedad acerca de los profesores. Esto explicaría, en cierto modo, por qué los alumnos más talentosos no estarían escogiendo las carreras de educación ${ }^{2}$. Por su parte, los programas de educación de las universidades chilenas no logran mejorar el desempeño de sus alumnos ni entregar un valor agregado (Manzi, Lacerna, Meckes y Ramos, 2011).

Respecto de las condiciones de trabajo, es posible observar que los salarios de los profesores son los más bajos en comparación con otras carreras profesionales en Chile (Mideplan, 2011) y, en relación con los otros países miembros de la OECD, las remuneraciones docentes están entre las más bajas y con escasa proyección de crecimiento (OECD, 2014). Junto con el reconocimiento, aspectos asociados a las condiciones de enseñanza dificultan aún más la labor de los docentes, como es el escaso tiempo no lectivo con que cuentan durante su jornada laboral.

2 Según los resultados del proceso de selección de universidades del CRUCH, en el 2014 solo el 14,4\% de los alumnos seleccionados en carreras de Educación obtuvo 650 puntos o más en el promedio de Lenguaje y Matemática de la PSU. 
Si bien en los últimos años se han producido cambios institucionales en Chile que afectan directa o indirectamente ciertos aspectos de la profesión ${ }^{3}$, así como diversas iniciativas y propuestas que buscan establecer formalmente una carrera docente ${ }^{4}$, no se ha concretado una política docente integral que favorezca la articulación del sistema educativo en función del desarrollo de los profesores y del mejoramiento de sus condiciones de enseñanza.

Sumado al diagnóstico respecto de la situación docente en Chile, a principios de 2014 el país también estaba viviendo cambios políticos y proyectando reformas nacionales. La literatura es clara en señalar que los momentos de reformas son siempre espacios donde los diferentes actores buscan poner sus intereses para lograr que las nuevas políticas públicas se basen en las creencias y medidas que dichos actores desean (Sabatier \& Wible, 2007). En este sentido, el anuncio de una nueva reforma tributaria, junto con el hecho de que una parte importante del dinero recaudado iba a ser destinado a financiar políticas públicas educativas, creó un espacio para que diferentes actores mostrasen su preocupación en torno a cómo se iban a gastar dichos recursos.

En este contexto nace, a fines de 2013, la idea de comenzar a trabajar en una propuesta que congregara a diversos actores e incorporara todos los aspectos y etapas de la profesión docente (desde la atracción hasta el retiro) y que permitiera, con un diagnóstico compartido, encontrar un espacio para mejorar las condiciones de enseñanza actuales y potenciar el desarrollo profesional de los profesores. Así, surgen los primeros lineamientos de lo que después se constituyó como "El Plan Maestro" (EPM). Este espacio de participación ciudadana se caracteriza por reunir diversos saberes,

3 Algunos ejemplos de estos cambios institucionales en Chile son: Ley General de Educación, Ley de Subvención Escolar Preferencial, sistema de aseguramiento de la calidad, Ley de Calidad y Equidad para la Educación, la beca Vocación de Profesor, convenios de desempeño con universidades y diversos incentivos monetarios a los docentes como el SNED, AVDI y AEP.

4 Entre estas iniciativas se destacan en los últimos años: las orientaciones entregadas por el Consejo Asesor Presidencial (2006), el Panel de Expertos (2010), la propuesta elaborada por la Comisión de trabajo entre Mineduc y el Colegio de Profesores (2008-2009), la propuesta coordinada por el Centro de Políticas Públicas UC y Elige Educar (2012) y el proyecto de ley presentado por el presidente Sebastián Piñera (2012). 
124 El PLAN MAESTRO, DiÁlogos PARA LA PROFESIÓN DOCENTE - H. Hochschild, F. Díaz, J. Walker, J. Schiappacasse y M. Medeiros

trayectorias, experiencias vitales y visiones acerca de la profesión docente, con el propósito de generar un diagnóstico y una visión común de los cambios que se necesitaban para una nueva política docente a partir del diálogo horizontal, colaborativo y transparente de todos quienes participaran de sus distintas instancias.

\section{El Plan Maestro (EPM): una plataforma de participación para dialogar respecto de la profesión docente}

El Plan Maestro nace como una plataforma de participación, con el fin de levantar reflexiones y propuestas concretas y, de esa forma, influir en el accionar gubernamental para que las políticas docentes tomaran un lugar importante entre las prioridades legislativas del gobierno. Actúa entonces como un grupo de presión donde:

"las personas que provienen de una variedad de posiciones (...), quienes comparten un particular sistema de creencia y que coordinan su comportamiento de diversas maneras, procuran manipular las reglas, presupuestos y el personal de las instituciones gubernamentales para alcanzar sus objetivos con el tiempo" (Sabatier y Jenkins-Smith, 1999, p. 5)

El núcleo aglutinador de los diferentes actores dentro de esta plataforma es la creencia común de que:

"para favorecer el pleno desarrollo de la persona humana en las distintas etapas de la vida (...) es necesario que las educadoras y educadores sean actores clave y que será imposible mejorar la calidad y equidad de nuestra educación si no mejoramos las condiciones de formación y ejercicio de la profesión de nuestras maestras y maestros, actuales y futuros, así como su relación con los otros miembros de la comunidad educativa" (El Plan Maestro, 2014, p. 8).

Sobre la base de esa creencia, la constitución y el accionar de EPM se asientan en un profundo diagnóstico acerca de la realidad política y social del país: por una parte, existen escasos incentivos políticos para dar prioridad al tema docente y, por otra, no existe un trabajo coordinado y sistemático entre los diferentes actores sociales y académicos interesados en avanzar en los temas docentes. 
Con el fin de dar prioridad política al tema docente se llegó a la conclusión de que para revertir esta situación se debía trabajar en la articulación comunicacional, técnica y estratégica de todos los actores pro políticas docentes.

Otro elemento que incentivó la creación de EPM fue la necesidad de alcanzar un consenso técnico lo que, según la literatura, era factible de lograrse a través de la consolidación de las relaciones y visiones de los diferentes actores docentes, con una coordinación y estrategia conjunta (Gómez, 2012), esto para poder influir en el espacio político con mayor fuerza.

Desde estos actores no existía una capacidad para coordinar esfuerzos y presionar al sistema político respecto de las temáticas docentes. Esto puede verse, por ejemplo, en la falta de un relato común entre los actores pro docentes en las comisiones del Congreso, donde estaban de acuerdo con avanzar en una carrera profesional docente, sin embargo, temas como el estatuto docente volvieron a ser trabas para lograr un consenso unánime.

Asimismo es posible observar que durante todos los procesos de reforma realizados durante 1990 y 2010, no se encuentran posturas unitarias entre los diferentes actores pro docentes, existiendo más espacio de competencia que de coordinación (Mizala, 2007).

Frente a esta realidad es que diversas instituciones -desde actores gremiales como sostenedores, universidades, profesores, estudiantes universitarios, estudiantes escolares hasta actores académicos como los centros de estudios, fundaciones, ONGiniciaron este proceso de convergencia.

Para poder desarrollar un proceso que creara confianza entre los diferentes actores y pudiese hacer notar las creencias comunes de esta coalición de causa, se desarrolló una metodología basada en los procesos de gestión de controversia. Esta metodología tiene como objetivo realzar los puntos comunes y sistematizar las divergencias, pero por sobre todo busca establecer espacios de trabajo en conjunto para la creación de una identidad pro docente. 


\section{El proceso detrás de El Plan Maestro}

El primer paso para la construcción de EPM fue invitar directamente a cada una de las instituciones y actores comprometidos con la educación a sumarse a este proceso. La invitación pretendió conformar un espacio de participación ciudadana que buscara sentar las bases de la política docente que se requiere desarrollar en Chile e invitara a priorizar el tema docente en la agenda política y pública. Así, se sumaron 20 instituciones, las que constituyeron la primera instancia de participación: el Consejo Consultivo ${ }^{5}$. Dicho Consejo fue el responsable de convocar y guiar el proceso y tuvo a su vez una Secretaría Técnica ${ }^{6}$, que se encargó de vincular los espacios de participación y coordinar y gestionar las reuniones de trabajo y, en general, ejecutar las decisiones del Consejo.

Otro espacio de participación fue el Foro Base, compuesto por más de 40 personas de todo Chile, representantes de diversas realidades del sistema educativo: maestras y maestros, asistentes de la educación, estudiantes, apoderados, académicos, directivos y sostenedores que provenían del norte y del sur, de sectores urbanos y rurales, hombres y mujeres de distintos niveles y especialidades de educación. El Foro Base, como principal órgano de participación, generó a través de acuerdos el contenido de las propuestas que serían la base de una nueva profesión docente, identificando a su vez las divergencias existentes entre sus miembros.

5 Las instituciones participantes de EPM son: Asociación Chilena de Municipalidades (AChM), Centro de Estudios de Políticas y Prácticas en la Educación (CEPPE), Centro de Estudios Públicos (CEP), Centro de Investigación Avanzada en Educación (CIAE), Centro de Investigación y Desarrollo de la Educación (CIDE), Centro de Políticas Comparadas de la Educación (CPCE), Colegio de Educadores de Párvulos de Chile A.G., Colegio de Profesores de Chile A.G., Colegios Particulares de Chile A.G. (CONACEP), Confederación de Estudiantes de Chile (CONFECH), Consejo de Decanos de la Educación del CRUCH, Consejo Nacional de Asistentes de la Educación, Coordinadora Nacional de Estudiantes Secundarios (CONES), Educación 2020, Elige Educar, Federación de Instituciones de Educación Particular (FIDE), Fundación Chile, Universidad Metropolitana de Ciencias de la Educación (UMCE), Vicaría para la Educación de Santiago. La iniciativa cuenta, además, con el apoyo de Unesco, oficina de Santiago, y de la Biblioteca Nacional de Santiago.

6 Integrada por Elige Educar, Educación 2020, Colegio de Profesores A.G. y la Vicaría para la Educación de Santiago. 
Por otro lado, para alimentar el debate y legitimar la discusión, se realizaron dos grandes Encuentros Ciudadanos en Antofagasta y Temuco, y más de 40 Encuentros Ciudadanos a lo largo de todo Chile, organizados por las propias comunidades educativas. Esto fue posible gracias a la utilización de un set de participación descargable de la página web de El Plan Maestro (elplanmaestro.cl). Así, cualquier comunidad interesada en discutir acerca de la profesión docente pudo generar sus propias propuestas y ser parte de la iniciativa. Todas fueron discutidas en la última sesión del Foro Base, siendo varias de ellas incorporadas en el documento final. La realización de los Encuentros Ciudadanos permitió recoger de mejor manera las diversas realidades educacionales y culturales de nuestro país.

\section{Gráfico 1: Modelo de participación interactivo de El Plan Maestro}

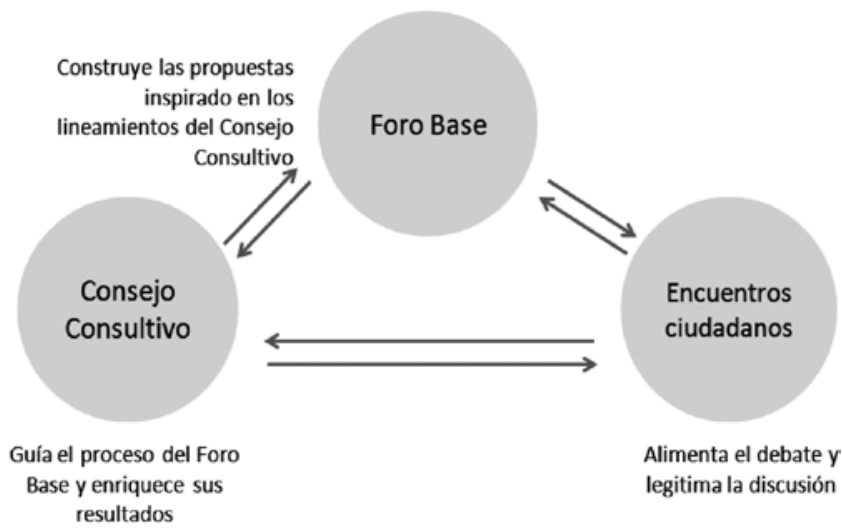

El Plan Maestro también generó distintas acciones públicas e intervenciones ciudadanas con el fin de llamar la atención acerca de lo importantes que son las maestras y maestros en el proceso educativo de los niños, niñas y jóvenes. Por ello, el set de participación mencionado anteriormente incluyó un conjunto de letras gigantes que permitieron a cientos de personas, desde Arica hasta Magallanes, hacer sus propias intervenciones públicas, para remecer e invitar a sus comunidades a reflexionar en torno a esta materia ${ }^{7}$.

$7 \quad$ El set de participación y las intervenciones urbanas fueron diseñados y coordinados por Mil M2, un centro cultural de innovación y participación ciudadana. 
128 El PLAN MAESTRO, DiÁlOGOS PARA LA PROFESIÓN DOCENTE - H. Hochschild, F. Díaz, J. Walker, J. Schiappacasse y M. Medeiros

Por último, como parte final del proceso, las propuestas y principios de EPM se hicieron públicas, invitando a la ciudadanía a sumarse a ellas a través de la campaña "Firme por la profesión docente". En esta se convocó a las personas y las organizaciones a adherirse a los principios que inspiran las propuestas, para así dar más fuerza ciudadana al proceso. El resultado consistió en más de 26.000 firmas de apoyo, que reflejaron el respaldo transversal a EPM.

\section{La metodología detrás de las instancias de participación}

El Plan Maestro adoptó diversos valores que se consideran fundamentales para la creación de una propuesta participativa, los cuales permitieron fortalecer el desarrollo del proceso:

- La apertura, comprendida como la convocatoria amplia a instituciones y personas, unidas por el interés de mejorar la profesión docente en Chile.

- El respeto, como exigencia de considerar al otro un interlocutor válido, en tanto condición para desarrollar un diálogo constructivo.

- La diversidad, entendida como el reconocimiento de la riqueza existente en los saberes, trayectorias, experiencias vitales y visiones referidas a la profesión docente.

- La transparencia, como la apertura al diálogo de la comunidad, dando libre acceso a la discusión a través de actas disponibles en un sitio web.

- El compromiso, como un esfuerzo compartido por alcanzar acuerdos totales, reconociendo que existen temas respecto de los cuales las posiciones son divergentes.

El trabajo en conjunto y constante del Foro Base y el Consejo Consultivo fueron fundamentales para la construcción de las propuestas. A lo largo del proceso se trabajó de manera coordinada en torno a cinco ejes temáticos:

- ¿Qué educadoras y educadores necesita Chile?

- ¿Cómo seleccionar y formar a los docentes que Chile necesita?

- ¿Cuáles son los elementos indispensables de la carrera docente y cómo debiera ser la evaluación docente? 
- ¿Cómo debieran ser las condiciones del ejercicio profesional y el desarrollo profesional docente?

- ¿Cómo debiera ser el rol de la comunidad educativa en los procesos de enseñanza y aprendizaje?

Para conversar y desarrollar las medidas, el Foro Base sesionó en seis ocasiones, entre el 2 de julio y el 10 de septiembre, en la Biblioteca Nacional de Santiago. En cada una de las sesiones se abordó un eje temático, quedando la sesión final para la síntesis y revisión de las propuestas, así como para la incorporación de los comentarios hechos por el Consejo Consultivo y las sugerencias emitidas desde los Encuentros Ciudadanos.

En cada sesión, la metodología de trabajo consistió en un plenario inicial donde los foristas tenían la posibilidad de entregar sus consideraciones iniciales acerca de la temática trabajada, para pasar luego a la discusión por grupos y por último a un plenario final en el cual se integraban las propuestas. Las medidas fueron sintetizadas por un grupo de escribanos en las actas de las sesiones (las que pueden encontrarse en la página web) y enviadas al Consejo Consultivo, que se encargó de pulir las propuestas y hacer comentarios de vuelta al Foro Base.

Junto con las propuestas surgidas de estas sesiones se presentan, a su vez, las divergencias. De esta manera, aparecen identificados aquellos aspectos respecto de los cuales no se alcanzó un acuerdo a lo largo del proceso, indicándose los principales argumentos que se contraponen, lo que puede contribuir también a la construcción de alternativas de política pública en educación.

El resultado del trabajo conjunto de todos los organismos e instancias de EPM está ordenado según los ejes temáticos que fueron discutidos en las sesiones que son, a su vez, las principales materias estimadas por el Consejo Consultivo para ser consideradas en una política nacional docente.

Así, gracias al trabajo sistemático y abierto de la sociedad civil organizada y a la adhesión de miles de ciudadanos, se pretende generar 
130 El PLAN MAESTRO, DiÁlOGOS PARA LA PROFESIÓN DOCENTE - H. Hochschild, F. Díaz, J. Walker, J. Schiappacasse y M. Medeiros

las bases para una nueva profesión docente, dando a esta carrera la valoración social que merece y facilitar de este modo el desarrollo pleno de millones de niños, niñas y jóvenes.

\section{Propuestas y principios orientadores generados para la política docente}

El resultado concreto del proceso fue la creación de 130 propuestas orientadas a entregar soluciones a los componentes y áreas que más afectan a los docentes y su ejercicio ${ }^{8}$. Las propuestas rescatadas dan cuenta de los temas que se consideran centrales para la enseñanza y la formación escolar, abordando el proceso desde que un joven ingresa a estudiar una carrera de educación, pasando por su proceso de formación y llegando a los aspectos necesarios de considerar para su ejercicio y desarrollo profesional.

Detrás de la discusión siempre estuvo presente la premisa de que la educación es un derecho y tiene por objeto el pleno desarrollo de la persona humana en las distintas etapas de su vida. Solo en la medida en que mejoren las condiciones de formación y ejercicio de todos los educadores y educadoras, será posible lograr calidad y equidad en nuestra educación.

Las 130 propuestas fueron traducidas en 12 principios que buscan orientar y reflejar los puntos prioritarios que debiera contener una nueva política nacional docente. Las propuestas y principios de EPM incluyen a todas las educadoras y educadores de enseñanza básica, enseñanza media de todas las especialidades, educación científico humanista y técnico profesional, diferenciales y de párvulos que se desempeñan en establecimientos educativos de todas las dependencias. A su vez, ciertos principios y propuestas hacen referencia a los asistentes de la educación, quienes también cumplen un rol fundamental en las comunidades educativas. Los doce principios son los siguientes:

8 El documento que sistematiza las 130 propuestas generadas por El Plan Maestro pueden encontrarse en el siguiente link: http://www.elplanmaestro.cl/download/ planmaestro\%20pagweb.pdf 
- Docentes con vocación para enseñar y un sólido manejo disciplinar y pedagógico. Chile necesita que estos docentes sean autónomos, empoderados, reflexivos, conscientes de su responsabilidad en la formación integral de ciudadanos y con altas expectativas respecto de la capacidad de aprender de sus estudiantes.

- Mejor formación inicial docente, con más exigencias y mayor vinculación con la realidad escolar. En todo Chile se requieren instituciones formadoras de alta calidad, selectivas en su ingreso, académicamente exigentes y orientadas a formar docentes vinculados con la realidad del aula y su contexto. El Estado debe resguardar que los diversos programas cumplan cabalmente con este propósito, así como promover una efectiva regulación de la oferta de carreras de Educación en el país. A su vez, motivará la inducción de los docentes que recién comienzan a ejercer la profesión, para acompañarlos en su primera etapa laboral.

- Carrera docente para los maestros de todos los establecimientos que reciben financiamiento público. La carrera docente debe servir de marco para todas las maestras y maestros que se desempeñen en instituciones que reciban recursos públicos, sin importar su dependencia.

- Carrera docente financiada por el Estado. La carrera docente debe estar financiada por el Estado en igualdad de condiciones, de modo que sea aplicable para todos los docentes de los establecimientos que reciben recursos públicos.

- Carrera docente ascendente y atractiva. La carrera docente debe tener etapas claras de progresión que consideren los momentos de inicio, desarrollo y retiro. La progresión dentro de ella tiene que ser ascendente en términos de remuneraciones y otros reconocimientos asociados, y fomentar que los buenos docentes de aula permanezcan en ella, promoviendo al mismo tiempo trayectorias profesionales alternativas. Las oportunidades de progresión dependerán de su desempeño, dentro y fuera de la sala de clases, y se considerará también la experiencia y el desarrollo profesional. La desvinculación laboral de los docentes, cuando corresponda, se concretará a través de mecanismos objetivos, públicos y transparentes, considerando oportunidades previas de mejoramiento. 
- Evaluación docente universal y formativa. La evaluación docente debe ser una exigencia para todos los docentes que trabajen en instituciones que reciben recursos públicos, y estar gobernada por un propósito eminentemente formativo. En tal sentido, y aun cuando puede tener consecuencias para el desarrollo de la carrera, su propósito primordial debe ser contribuir al proceso educativo y a la mejora continua, considerando siempre el contexto en el que se desarrolla la labor docente.

- Desarrollo profesional docente para mejores logros educativos. El desarrollo profesional debe poner el foco en el aula y privilegiar la calidad del aprendizaje. Esto exige que los programas de formación se orienten a mejorar prácticas, competencias y habilidades pedagógicas y didácticas.

- Mejora sustancial de remuneraciones para actuales y futuros docentes. Es necesario aumentar sustancialmente las remuneraciones de todas las maestras y maestros, para que el reconocimiento que reciben por su trabajo esté en relación con la complejidad de su labor y la responsabilidad que recae sobre ellos.

- Mejores docentes para los sectores de mayor vulnerabilidad. Todos los niños, niñas y jóvenes de Chile tienen derecho a contar con grandes profesoras y profesores en sus salas de clases. Dado que vivimos en un país con alta segregación social, es en los sectores que concentran a los estudiantes con mayor vulnerabilidad donde se requieren las mejores educadoras y educadores. Por lo tanto, la carrera docente debe procurar formas efectivas de fomentar que ellos trabajen en dichos sectores.

- Aumento significativo y delimitación del uso de horas no lectivas. Es imprescindible aumentar significativamente la proporción de horas no lectivas, de forma que exista el tiempo adecuado para realizar labores tan importantes como la planificación, los procesos de evaluación, el trabajo colaborativo y la relación directa con la comunidad, entre otras dimensiones centrales del aprendizaje.

- Escuelas y jardines infantiles protagonistas de su desarrollo. Los establecimientos educacionales deben desempeñar un rol protagónico en el diseño y ejecución de sus propios planes institucionales, así como de sus programas de desarrollo profesional docente. Para esto último, mantendrán una articulación constante 
con el Estado, las universidades u otras entidades formativas de forma que cada institución sea efectivamente una comunidad de aprendizaje.

- Comunidad educativa integradora y colaborativa en el mejoramiento de los procesos educacionales y del aprendizaje. La carrera docente debe dialogar con una nueva visión de la comunidad educativa en la que los docentes, asistentes de la educación, directivos, sostenedores, apoderados y estudiantes se comprendan a sí mismos como un solo equipo, con el objetivo común de formar una comunidad educativa que brinde las mejores oportunidades de aprendizaje a los niños, niñas y jóvenes. En tal sentido, es necesario reconocer e integrar la riqueza de la diversidad, así como también reconocer el rol fundamental que cumplen los asistentes de la educación en este aspecto.

\section{Efectos en política pública docente}

Las propuestas y principios que surgieron de EPM pretenden ser insumos para la definición de las políticas públicas en torno a la materia, partiendo de la base que se necesita mejorar significativamente la manera en que en Chile se atrae, forma y retiene a los docentes, para así avanzar hacia una meta mayor: que cada niño, niña y joven cuente con profesores de excelencia, de tal manera que la educación sea un motor de desarrollo integral.

El proceso del EPM permitió que instituciones muy diversas dialogaran generando una propuesta común, demostrando que es posible avanzar si se construyen las confianzas necesarias, para lo cual es necesario transparentar posiciones y buscar un lenguaje común que permita acercar puntos de vista.

Este proceso de construcción de confianzas ha permitido abordar acciones conjuntas en función de la propuesta de EPM y hacer un frente común, respetando las legítimas diferencias frente al tema docente.

Los resultados, una vez finalizado el proceso de levantamiento de propuestas, fueron entregados al Ministro de Educación el 20 de 
134 El PLAN MAESTRO, DiÁlOGOS PARA LA PROFESIÓN DOCENTE - H. Hochschild, F. Díaz, J. Walker, J. Schiappacasse y M. Medeiros

octubre de 2014 y desde allí han sido un importante insumo para la formulación de una política nacional docente.

Desde el punto de vista del impacto, no solo se logró definir 130 propuestas de carácter unánime entre los diferentes participantes, sino que también se pudo dar relevancia mediática a través de la realización de diversos actos públicos, los cuales incluyen desde presentaciones a las comisiones de educación de la Cámara de Diputados, hasta la celebración de actividades culturales. En total, EPM tuvo una aparición de al menos 40 veces en medios de comunicación, durante el segundo semestre de 2014, eso sin contar las más de 26 mil personas que adhirieron a sus principios, comprometiéndose con una nueva política docente.

\section{Referencias}

El Plan Maestro (2014). Principios y propuestas de El Plan Maestro. Santiago. Recuperado el 2 de diciembre de 2014 desde http://www.elplanmaestro. cl/download/planmaestro\%20pagweb.pdf.

García, S., Maldonado, D., Perry, G., Rodríguez, C. \& Saavedra, J. E. (2013). Tras la excelencia docente: ¿cómo mejorar la calidad de la educación para todos los colombianos? Recuperado el 2 de diciembre de 2014 desde http://fundacioncompartir.org/front/media/Tras_la_excelencia_ docente_Resumen_Ejecutivo_Final.pdf

Gómez Lee, M. I. (2012). El marco de las coaliciones de causa [Advocacy Coalition Framework]. Revista Opera 12, 11-30.

Manzi, J., Lacerna, P., Meckes, L. y Ramos, I (2011). ¿Qué características de la formación inicial de los docentes se asocian a mayores avances en su aprendizaje de conocimientos disciplinarios? Informe final Fonide NF511015. Santiago: Ministerio de Educación.

Ministerio de Planificación de Chile, Mideplan (2011). Informe de politica social 2011. Santiago: Ministerio de Planificación.

Mizala, A. (2007). La economía política de la reforma educacional en Chile. Recuperado el 2 de diciembre del 2014 desde http://cieplan.cl/media/ publicaciones/archivos/153/Capitulo_1.pdf

Organización para la Cooperación y el Desarrollo Económicos, OECD (2005). Attracting, developing and retaining effective teachers: teachers matter. Paris, OECD 2005. 
Organización para la Cooperación y el Desarrollo Económicos, OCDE (2014). Education at a Glance 2014. OECD Indicators. Paris, OECD

Rivkin, S. G., Hanushek, E. A., \& Kain, J. F. (2005). Teachers, schools, and academic achievement. Econometrica, 73(2), 417-458.

Rockoff, J. E. (2004). The impact of individual teachers on student achievement: evidence from panel data. American Economic Review, 94, 247-252.

Sabatier, P. A., \& Jenkins-Smith, H. C. (1999). The advocacy coalition framework: an assessment. Theories of the policy process, 118, 188.

Sabatier, P., \& Weible, C. (2007). The advocacy coalition: innovations and clarifications.

En P. A. Sabatier (ed.), Theories of the policy process. Boulder: Westview Press, pp. 189-222.

Sanders, W.L. \& Rivers, J. C. (1996). Cumulative and residual effects of teachers on future student academic achievement. Knoxville: University of Tennessee Value-Added Research and Assessment Center.

Recibido: 12/12/2014

Aceptado: 15/12/2014 